Rev. Elev. Méd. vét. Pays trop., 1971, 24 (2): 183-89

\title{
Etat immunitaire actuel, naturel ou acquis du cheptel sénégalais vis-à-vis de la peste bovine, de la maladie des muqueuses, de la rhinotrachéite infectieuse et de la maladie respiratoire à virus Parainfluenza III
}

\author{
par G. BERNARD et P. BOURDIN
}

\begin{abstract}
RESUME
Les auteurs ont contrôlé par des techniques sérologiques appropriées l'immunité naturelle ou acquise des bovins, ovins et caprins sénégalais vis-à-vis des maladies contagıeuses suivantes: la peste bovine, la maladie des muqueuses, la rhınotrachéite infectieuse et l'affection respiratoire à parainfluenza III.

En ce qui concerne la peste bovine, après les trois années de la campagne conjointe de vaccination des bovins, le taux d'animaux immunisés est égal à $B 0$ p. 100 . Un contrôle fait chez les petits ruminants montre que 50 p. 100 des moutons et des chèvres ont à l'état naturel des anticorps. neutralisant le virus pestique. Pour la maladie des muqueuses et la maladie respiratoire à Parainfluenza III, 50 p. 100 des bovidés et des petits ruminants ont des anticorps, Quant à la rhinotrachéite infectieuse, seuls les bovidés ont des anticorps.
\end{abstract}

\section{INTRODUCTION}

Les états du Centre et de l'Ouest Africain, situés dans la zone sahélienne ont une économie rurale en grande partie basée sur l'élevage. Pour protéger leur cheptel, ils ont entrepris, sous l'égide de la Commission Scientifique, Technique et de la Recherche, une campagne conjointe de lutte contre la peste bovine, financée par la communauté économique européenne et les Etats-Unis.

Au Sénégal, malgré les campagnes annuelles de vaccination entreprises par le Service de l'Elevage, la peste bovine était régulièrement signalée chaque année surtout dans les régions de transhumance.

La mise en œuvre de la campagne conjointe de vaccination contre la peste bovine pour
l'Ouest Africain à partir de 1966, s'est traduite par une diminution du nombre des foyers, et la disparition de la maladie en 1967. L'enquête sérologique effectuée au cours de cette campagne a pour but de vérifier si la diminution des cas de peste bovine est en rapport avec une augmentation du pourcentage des animaux immunisés.

Cette enquête a été étendue aux petits ruminants, ceux-ci pouvant, soit, comme l'ont constaté ZWART et MACADAM (1967), dans de très rares cas héberger le virus pestique virulent et le transmettre aux bovins, soit le plus souvent, du moins au Sénégal, faire une affection spécifique, décrite par MORNET, ORUE et collab. (1956) sous le nom de peste des petits ruminants. Cette maladie est due, comme l'ont montré GILBERT et MONNIER (1962) 
BOURDIN et LAURENT-VAUTIER (1967), à un mutant du virus pestique adapté aux petits ruminants.

En outre, la vaccination généralisée du cheptel en entraînant la disparition de la peste bovine peut révéler la présence de maladies apparentées qui jusqu'ici ont pu être confondues avec elle, ainsi que l'ont montré, en Afrique Centrale, PROVOST BORREDON et FEREOL (1964), PROVOST, BOGEL, BORREDON et MAURICE (1967) et PROVOST BORREDON, QUEVAL et MAURICE (1967).

Parmi ces affections, il faut citer par ordre de gravité décroissante la maladie des muqueuses, la rhinotrachéite bovine infectieuse et les maladies dues à des virus Parainfluenza.

Pour confirmer l'existence au Sénégal de ces maladies apparentées à la peste bovine et connaître leur importance, de nombreux échantillons de sérums ont été examinés en vue de rechercher la trace d'une affection apparente ou non.

\section{MATERIEL ET METHODES}

Il a été nécessaire d'utiliser des procédés différents d'étude adaptés à chaque virus.

\section{La séro-neutralisation}

En ce qui concerne les virus de la peste bovine (PB) et de la peste des petits ruminants (PPR) la réaction de séro-neutralisation classique décrite par PLOWRIGHT et FERRIS (1961) a été utilisée. Puis pour des raisons de commodité, il a été fait une adaptation de la méthode cinétique de séro-neutralisation mise au point par LEPINE, ROGER et ROGER (1959) pour la recherche des anticorps neutralisant la poliomyélite.

L'immunité du troupeau vis-à-vis de la maladie des muqueuses (MM) et de la rhinotrachéite bovine infectieuse (RBI) a été contrôlée par des épreuves de séro-neutralisations cinétiques adaptées à ces espèces virales.

Nous ne reviendrons pas aux détails de la méthode classique de séro-neutralisation, ni sur l'adaptation de la séro-neutralisation cinétique du virus $P B$, décrite par BOURDIN et BERNARD (1967).
Pour la recherche des anticorps neutralisant le virus PB dans les sérums bovins et celle des anticorps neutralisant le virus PPR dans les sérums ovins et caprins, seul le virus $\mathrm{PB}$ a été utilisé en partant du fait que les virus PB et PPR ont des rapports antigéniques très étroits.

Autre point à préciser, pour la recherche des anticorps neutralisant les virus MM et RBI, il n'a pas été ajouté de soude décinormale et de bicarbonate de sodium en dehors de la quantité normale de bicarbonate contenue dans le milieu de Hanks LAYE.

Les temps de lectures sont fixés au $4^{\mathrm{e}}$ jour pour le virus $\mathrm{MM}$ et au $3^{\mathrm{c}}$ pour le virus RBI.

\section{L'hémagglutination et son inhibition}

Nous avons utilisé cette méthode pour la recherche des anticorps antivirus parainfluenza, grâce à une adaptation de l'épreuve standard d'inhibition de l'hémagglutination pour le diagnostic de la grippe (O.M.S., 1959).

\section{A. Les réactifs en présence}

a) Les milieux : on emploie comme milieu de dilution et de suspension des hématies, du sérum physiologique à 8,5 p. 1.000 . D'autre part le sang du mouton est recueilli dans du citrate de soude à 2 p. 100 dans du sérum physiologique.

b) Les hématies de mouton: le sang est recueilli dans un tube contenant 20 p. 100 de citrate de sodium puis est lavé et centrifugé, le culot globulaire est recueilli et conservé à +4 " C. Au moment de leur utilisation les hématies sont remises en suspension à la concentration de 0,5 p. 100.

La conservation du culot étant limité l'utilisation des hématies fraî́ches a été remplacée par celle des hématies formolées selon la méthode préconisée par FLICK (1948) et FAUCONNIER (1958-1959).

Ici le culot globulaire est repris dans du sérum physiologique tamponné auquel on ajoute 20 p. 100 d'une solution au demi de formol à 30 p. 100 . On agite et l'on met au bain-marie à $37^{\circ} \mathrm{C}$ pendant une heure. Les hématies sont alors lavées trois fois puis on recommence l'opération avec le formol successivement trois fois.

Le culot du dernier lavage est remis en suspension à 25 p. 100 dans du sérum physio- 
logique tamponné. On ajoute 0,3 p. 100 de formol et l'on répartit en flacons bouchés. La conservation de cette suspension est de plusieurs mois à $+4^{\circ} \mathrm{C}$.

Lors de l'emploi, la concentration en hématies qui était de $0,5 \mathrm{p}$. 100 pour la $1^{\text {re }}$ méthode devra être portée ici à 1 p. 100.

c) Le virus ou antigène: on utilise une souche adaptée aux cellules de $1^{\text {re }}$ explantation de rein d'embryon de veau dans un milieu nutritif de HANKS LAYE avec sérum de cheval. Au $6^{\mathrm{e}}$ jour de culture, la suspension virulente est mise au congélateur.

d) Sérums à tester : comme pour les autres méthodes, les sérums sont recueillis sur les lieux mêmes d'habitat des troupeaux. Les sangs prélevés par ponction de la veine jugulaire sont centrifugés sur place puis récoltés et acheminés sous glace au laboratoire où ils sont décomplémentés 30 minutes à $56^{\circ} \mathrm{C}$ et conservés à $-20^{\circ} \mathrm{C}$ en attendant leur emploi.

Ils ne subissent pas un traitement spécial afin d'éliminer les inhibiteurs non spécifiques car comme PROVOST et BORREDON (1965) l'ont fait remarquer, au-delà de $1 / 10$ ils sont trop dilués pour être perceptibles. Or pour la réaction la dilution des sérums est $1 / 40$.

\section{B. Titrage du virus}

On réalise des dilutions du virus du $1 / 2$ au $1 / 512$ dont on répartit $0,2 \mathrm{ml}$ dans des capsules d'une plaque en matière plastique type SALK (1948). On ajoute $0,2 \mathrm{ml}$ d'une suspension d'hématies formolées à 1 p. 100. Après agitation on place la plaque à $+4^{\circ} \mathrm{C}$ pendant trois heures.

On détermine alors le point terminal de l'hémagglutination partielle (50 p. 100) donné par la dilution suivant la dernière où ii $\mathrm{y}$ a eu agglutination complète. Lors de la réaction de I'inhibition de l'hémagglutination, la suspension virale contient 8 unités hémagglutinantes 50 p. 100 pour $0,2 \mathrm{ml}$.

\section{La réaction d'inhibition de l'hémagglutination}

Les sérums sont dilués au $1 / 40,0,2 \mathrm{ml}$ sont placés dans une capsule de la plaque puis on ajoute $0,2 \mathrm{ml}$ de l'antigène convenablement dilué et $0,2 \mathrm{ml}$ d'une suspension d'hématies.

Après agitation la plaque est mise 3 heures à $+4^{\circ} \mathrm{C}$, après ce délai on procède à la lecture.

Dans certaines cupules où il y a inhibition de l'hémagglutination le sérum correspondant possède des anticorps au $1 / 40$.

Dans d'autres il y a agglutination : le sérum correspondant ne possède pas d'anticorps au $1 / 40$.

\section{RESULTATS}

7.487 examens sérologiques ont été réalisés, répartis ainsi :

- Recherche des anticorps anti-PB chez les bovins : 969 .

- Recherche des anticorps anti-PB chez les petits ruminants : 1.576 .

- Recherche des anticorps anti-PI : 2.325.

- Recherche des anticorps anti-RBI : 1266.

Il s'est avéré plus simple de grouper l'ensemble des résultats dans un tableau unique. Il convient de rappeler que les résultats sont uniquement d'ordre qualitatif et sont obtenus sur des sérums dilués au $1 / 10$ pour la recherche des anticorps neutralisant les virus pestiques,

\begin{tabular}{|c|c|c|c|c|c|c|c|c|c|c|c|c|}
\hline & \multicolumn{3}{|c|}{$\begin{array}{c}\text { Anticorps } \\
\text { ant1-pestiques } \\
\text { P. } 100\end{array}$} & \multicolumn{3}{|c|}{$\begin{array}{c}\text { Anticorps } \\
\text { anti-paralnfluenza } \\
\text { p. } 100\end{array}$} & \multicolumn{3}{|c|}{$\begin{array}{c}\text { Anticorps } \\
\text { ant1-maladie des } \\
\text { muqueuses } \\
\text { p. } 100\end{array}$} & \multicolumn{3}{|c|}{$\begin{array}{c}\text { Anticorps } \\
\text { anti-rhinotrachéite } \\
\text { p. } 100\end{array}$} \\
\hline & Bovins & Ovins & Caprins & Bovins & ovins & Caprins & Bovins & Ovins & Caprine & Bovins & Ovins & Caprins \\
\hline Cap-Vert & & 62 & 45 & & 47 & 51 & & 55 & 49 & & 10 & 7 \\
\hline Sénégal orlental & 63 & 35 & 27 & & & 21 & 78 & & 68 & 38 & & 7 \\
\hline Casamance & 60 & 47 & 60 & 58 & 47 & 61 & 61 & & & 61 & & \\
\hline Fleuve & 69 & & & 25 & & & & & & & & \\
\hline Ferlo & BO & 45 & 66 & 37 & 23 & 28 & 67 & 41 & 60 & 48 & 0 & 0 \\
\hline
\end{tabular}


les virus de la maladie des muqueuses et de la rhinotrachéite. Pour les anticorps inhibant l'hémagglutination due au virus Parainfluenza la dilution est au $1 / 40$. Les pourcentages indiqués dans le tableau correspondent à des pourcentages de présence d'anticorps dans des échantillons de sérums prélevés dans une région donnée.

\section{DISCUSSION}

Les enquêtes sérologiques révèlent un certain nombre de faits dont l'interprétation permet de dégager quelques conclusions relatives à la peste bovine, la peste des petits ruminants et les maladies apparentées.

\section{A. Anticorps anti-pestiques chez les bovins}

On ne connaît pas les pourcentages d'anticorps avant vaccination. On peut seulement dire qu'après la $1^{\text {re }}$ année de vaccination 60 p. 100 du cheptel est immunisé, 70 p. 100 après la deuxième année, 80 p. 100 après la troisième.

Classiquement il est admis qu'un pourcentage d'immunisation de 70 à 75 p. 100 dans une population vis-à-vis d'une maladie contagieuse est suffisant pour protéger cette population. Il apparaît donc que la population bovine sénégalaise est actuellement protégée contre la peste bovine.

Des études sérologiques réalisées au Nigéria (1965) donnent un pourcentage d'animaux plus élevé après la $3^{\mathrm{e}}$ année de vaccination; il convient de préciser que les sérums des animaux nigérians étaient examinés après une dilution au $1 / 2$.

Si l'on examine les pourcentages d'animaux immunisés en fonction de l'âge, on constate, ce qui a déjà été observé au Nigéria, que les pourcentages sont plus faibles parmi les jeunes que parmi les adultes.

En ce qui concerne l'étude qualitative, la dilution des sérums au 1/10 a été choisie car elle est le témoin d'une immunité valable suivant PLOWRIGHT et FERRIS (1961).

Des tests quantitatifs effectués au Laboratoire d'Elevage de Dakar (1968) ont montré que les taux neutralisants les plus courants se situent au niveau du $1 / 40$.
La campagne terminée, cet état immunitaire ne sera maintenu que si des mesures sanitaires et conservatoires sont rigoureusement appliquées.

\section{B. Anticorps anti-bovipestiques chez les petits ruminants}

On peut conclure, du moins au Sénégal, d'après les différents résultats, qu'en zone d'endémie de peste bovine les anticorps existent chez 45 à 65 p. 100 des ovins et des caprins.

En zone indemne, seulement 25 à 35 p. 100 des animaux possèdent des anticorps.

\section{Anticorps anti-parainfluenza}

Ces affections pouvant présenter des symptômes apparentés à la $\mathrm{PB}$, il convient de savoir si les bovins possèdent des anticorps signalant leur existence.

\section{a) Présence d'anticorps chez les bovins}

Les pourcentages d'animaux ayant des anticorps se situent entre 25 et 58 p. 100 , les plus forts pourcentages pouvant coïncider avec des foyers latents. PROVOST en 1967, au Tchad, obtient des chiffres beaucoup plus élevés et explique ce fait par l'importance des rassemblements d'animaux qui sont moindres au Sénégal.

D'autre part, les pourcentages semblent équivalents dans toutes les catégories, les adultes pouvant être immunisés plus facilement (la maladie frappe surtout les jeunes veaux).

Des essais quantitatifs ont révélé que les anticorps pouvaient exister jusqu'à une dilution de 1/160 mais cela ne prouve pas que l'immunité des bovins soit suffisante. On peut uniquement dire qu'ils ont été en contact avec le virus.

Dans les mesures qui pourraient être envisagées en cas d'aggravation de la maladie, il semblerait que les grands rassemblements soient à éviter. Il conviendrait également de rechercher un vaccin à appliquer surtout aux jeunes animaux.

\section{b) Présence d'anticorps} chez les petits ruminants

Les petits ruminants n'extériorisant pas d'affection, il convenait de voir s'ils n'étaient pas susceptibles au moins d'héberger le virus. 
Les pourcentages d'animaux ayant des anticorps se situent entre 21 et 61 p. 100 . Les caprins semblent légèrement plus sensibles. Il n'y a pas de différences flagrantes entre les catégories d'âge. On peut penser qu'il y a une similitude entre ces résultats et ceux obtenus chez les bovins.

Il ne semble pas que l'affection soit reconnue chez les petits ruminants. Ils représentent un danger latent pour les bovins mais la séparation des deux espèces n'est pas possible dans l'élevage traditionnel.

\section{Anticorps anti-MM}

La maladie existe en Afrique comme l'ont montré BROWN et SCOTT (1957) et PROVOST et collab. (1967). Il convenait de vérifier au Sénégal son incidence dans les troupeaux.

\section{a) Présence d'anticorps chez les bovins}

Les pourcentages d'animaux présentant des anticorps se situent entre 61 et 78 p. 100 . Ces résultats sont voisins de ceux obtenus au Tchad par PROVOST en 1967. L'immunité naturelle semble assez importante. De par les résultats obtenus, on peut penser que la maladie sévit par périodes.

L'étude quantitative montre que l'immunité naturelle est très forte puisque les anticorps existent jusqu'à une dilution au 1/160.

La disparition de la peste bovine entraînera peut être une prophylaxie spécifique de cette maladie. Il existe deux vaccins : l'un de culture cellulaire, l'autre préparé à partir de rates de lapin infectées. Mais il faut prévoir surtout une surveillance attentive des troupeaux.

\section{b) Présence d'anticorps}

chez les petits ruminants

Le pourcentage d'animaux possédant des anticorps varie entre 41 et 68 p. 100. La sensibilité des petits ruminants semble donc assez importante mais ils n'extériorisent pas de maladie. Au point de vue régional, on retrouve de nouveau une similitude avec les bovins. On doit surtout envisager des mesures vis-à-vis des bovins, les petits ruminants pouvant les contaminer, mais ces risques seront toujours présents.

\section{E. Anticorps anti-RBI}

L'affection a été isolée en Afrique du Sud
(MARE et VAN RENBURG) (1961) en Côte d'Ivoire (NGUYEN-BA-VY) (1964) et au Tchad PROVOST et collab. (1964). Il convenait de vérifier si elle existait au Sénégal chez les bovins et les petits ruminants.

\section{a) Présence d'anticorps chez les bovins}

Les résultats concernant les pourcentages d'animaux ayant des anticorps sont semblables à ceux obtenus en Afrique Centrale. La race des animaux ne semble pas jouer quant à la sensibilité. Ce sont les plus jeunes qui ont les pourcentages les plus élevés.

Etude quantitative: Des études réalisées au laboratoire ont montré une sensibilité assez faible (les anticorps n'existent que rarement après une dilution au 1/20).

Mesures envisagées : à l'heure actuelle, l'affection paraît bénigne. Au cas où elle prendrait de l'extension, il existe des vaccins vivants et inactivés. Le point le plus important reste actuellement le diagnostic différentiel avec la peste bovine.

\section{b) Présence d'anticorps}

chez les petits ruminants

A la suite de notre étude sérologique, il ne semble pas que les petits ruminants présentent un danger de contagion pour les bovins au Sénégal puisque pratiquement réfractaires et insensibles à l'antigène viral (les anticorps lorsqu'ils existent ne sont présents au maximum que parmi 10 p. 100 de la population). Aucune mesure particulière n'est donc préconisée mais des vérifications épisodiques devront être effectuées afin de s'assurer de la continuité de ces résultats.

\section{CONCLUSION}

Les contrôles sérologiques sur un échantillonnage important de sérums ont été facilités par l'utilisation de la méthode cinétique et son adaptation au virus de la peste bovine, de la maladie des muqueuses et de la rhinotrachéite infectieuse. Les résultats font apparaître que vis-à-vis de la peste bovine, l'immunité du troupeau est solide; ces résultats se maintiendront si les mesures conservatoires sont rigoureusement appliquées.

Un problème annexe à la peste bovine est soulevé par la présence d'anticorps neutralisant le virus $\mathrm{PB}$ chez les petits ruminants. Il est 
vraisemblable de rattacher leur existence à la transmission du virus de la peste des petits ruminants, ce dernier étant un mutant du virus bovipestique. Il convient de poser la question du retour à sa virulence originelle.

L'étude immunologique des maladies appa- rentées à la peste bovine a révélé qu'elles existaient au Sénégal. Il est à craindre que, dans un proche avenir, ces maladies, dépouillées du contexte peste bovine, soient cliniquement signalées, le diagnostic différentiel devra alors intervenir.

\section{SUMMARY}

Natural or post-vaccinal present immunity of senegalese cattle sheep and goats for rinderpest, mucosal disease, infectious rhinotracheitis and parainfluenza III virus

The authors checked by appropriated serological technics natural and post-vaccinal immunity of senegalese cattle, sheep and goats for the following infectious diseases: rinderpest, mucosal disease, bovine infectious rhinotracheitis and respiratory disease caused by parainfluenza III virus.

As regard rinderpest, after three years of a joint campaign of vaccination of cattle, the rate of immune bovines is about 80 p. 100 . A survey made on small ruminants shows that 50 p. 100 of sheep and goats present neutralizing antibodies against rinderpest virus. As for mucosal disease and respiratory disease parainfluenza III virus, in 50 p. 100 of bovines and small ruminants antibodies can be detected. With respect to rhinotracheitis only bovines show antibodies.

\section{RESUMEN}

Estado de inmunidad actual, natural o adquirido del ganado senegalense enfrente de la peste bovina, de la enfermedad de las mucosas,

de la rinotraqueitis infecciosa y de la enfermedad respiratoria debida al virus Parainfluenza JII

Los autores han comprobado con tecnicas serologicas adaptadas la inmunidad natural o adquirida de los bovinos, los ovinos y los caprinos senegalenses enfrente de las enfermedades contagiosas siguientes: la peste bovina, la enfermedad de las mucosas, la rinotraqueitis infecciosa y la afeccion debida al virus parainfluenza IlI.

Para lo que concierne la peste bovina, despues de los tres años de la campaña conjunta de vacunación de los bovinos, la cantidad de animales inmunizados acerca los $80 \mathrm{p}, 100$. Una verificacion hecha entre los pequeños rumiantes demuestra que 50 p. 100 de los caprinos y de los ovinos tienen naturalmente anticuerpos neutralizando el virus pestico. Para la enfermedad de las mucosas y la enfermedad respiratoria debida al virus parainfluenza III, 50 p. 100 de los bovinos y de los pequeños rumiantes tienen anticuerpos. En cuanto a la rinotraqueitis infecciosa, los bovinos solamente tienen anticuerpos.

\section{BIBLIOGRAPHIE}

BOURDIN (P.) et BERNARD (G.) « Application de la méthode de séro-neutralisation cinétique à la recherche des anticorps neutralisant le virus de la peste bovine chez les bovins, les caprins et les ovins », Rev. Elev. Méd. vét. Pays trop., 1967, 20 (4) : 531-35.

BOURDIN (P.) et LAURENT-VAUTIER (A.), « Note sur la structure du virus de la peste des petits ruminants », Rev. Elev. Méd. vét. Pays trop. 1967, 20 (3) : 383-86.

BROWN (R. D.) et SCOTT (G. R.), « Mucosal disease complex », Vet. Rec., 1957, 69 (38) : 916.

FAUCONNIER (B.), «Utilisation des hématies hyper- formolées en virologie. I. Ann. Inst. Pasteur, 1958, 95, 777-80.

FAUCONNIER (B.), «Utilisation des hématies hyperformolées en virologie. II. Ann. Inst. Pasteur, $1959,96,110-13$

FLICK (J. A.), Proc. Soc. Exp. Biol., 1948, 68; 448.

GILBERT (Y.) et MONNIER (J.), «Adaptation d'une souche de virus bovipestique à la culture cellulaire. Premiers résultats ». Rev. Elev. Méd. vét. Pays trop., 1962, 15 (4) : 311-20.

Laboratoire de l'Elevage de Dakar-Hann, « Rapport annuel », 1968.

Laboratoire de Vom-Nigeria, * Rapport annuel», 1965. 
LEPINE (P.), ROGER et ROGER (A.) « La réaction cinétique de séroneutralisation des virus poliomyélitiques », Bull. OMS, 1959, 20, 563-78.

MARE (J.) et VAN RENSBURG (S. J.), " The isolation of viruses associated with infertility in cattle. A preliminary report », J.S. Afr. vet. Med. Ass., 1961, 32, 201-10.

MORNET (P.), et collab., « La peste des petits ruminants en Afrique Occidentale Française. Les rapports avec la peste bovine ", Rev. Eler. Méd. vét. Pays trop., 1956, 9 (4) : 313-42

NGUYEN BA-VY et PERREAU (P.) «Culture du virus de la rhinotrachéite infectieuse des bovins sur les cellules testiculaires d'embryon de mouton ", Rev. Elev. Méd. vét. Pays trop., 1964, 17 (2): 197-203.

Organısation Mondiale de la Santé, «Maladies à virus, des voies respiratoires. jer rapport du Comité d'experts \#, 1959. (Sér. Rapp. Techn. n* 170).

PLOWRIGHT (W.) et FERRIS (R. D.), "Stuc es with rinderpest virus in tissue culture. III. The stability of cultured virus and its use in virus neutralisation tests ». Arch. ges. Virus-forsch., 1961, 2 : 516-23.
PROVOST (A.) et collab., *La maladie des muqueuses en Afrique Centrale. Observations cliniques et épizootiologiques ", Rev. Elev. Méd. vét. Pays trop., 1967, 20 (1): 27-29.

PROVOST (A.) et BORREDON, « Rapport annuel de la région de recherches vétérinaires et zootechniques de l'Afrique Centrale $", 1965$, pp. 60-61.

PROVOST (A.), BORREDON (C.) et FEREOL (C.), « Note sur la rhinotrachéite infectieuse bovine en Afrique Centrale", Rev. Elev. Méd. vét. Pays trop., 1964, 17 (2) : 187-96.

PROVOST (A), et collab., «Enquête sur l'infection des bovidés par le virus parainfluenza 3 en Afrique Centrale. Application au contrôle de la sérologie de la péripneumonie », Rev. Elev. Méd. vét. Pays trop., 1967, 20 (1): 51-59.

SALK (J. E.), Science, 1948, 108, 749.

ZWART (D.) et ROWE (L. W), «The occurrence of rinderpest antibodies in the sera of sheep and goats in northern Nigeria ", Res. vet Sci., 1967, 7, 504-11. 